\title{
MODELLING A NEURON USING A CUSTOM MATH LIBRARY sfloat24 - IMPLEMENTATION OF A SIGMOID FUNCTION ON A FPGA DEVICE -
}

\author{
M. C. Miglionico \\ Department of Culture of the Project - Second University of Napoli \\ Via S. Lorenzo, Monastero di San Lorenzo, I-81031 Aversa (CE) - ITALY \\ BENECON Scarl - Member of UNESCO \\ E-mail: mcristina.miglionico@unina2.it \\ F. Parillo* \\ Department of Automation, Electromagnetism, Computer Science and Industrial Mathematics \\ University of Cassino, Via G. Di Biasio 43, I-03043 Cassino (FR) - ITALY \\ E-mail:f.parillo@unicas.it
}

\begin{abstract}
Artificial neuron networks base their processing capabilities in a parallel architecture. This makes them useful to solve pattern recognition, system identification and control problems. In this paper is described an artificial neuron implementation on a FPGA device by using a custom sfloat24 math library. Using this library have been implemented some activation functions of an artificial neuron. In particular this work discusses the analytical formulation of a sigmoid activation function and the consequent implementation on a FPGA device, using the custom built sfloat 24 math library.

The simulation results show that the sfloat 24 floating point math library and the implementation of an artificial neuron network are feasible, and the ir outperform by high-speed response.
\end{abstract}

Keywords: Artificial Neuron Networks (ANN), Field Programmable Gate Array (FPGA).

\section{Introduction}

Artificial Neuron Networks (ANN) are used with success in pattern recognition problems, function approximation, control, etc. Their processing capabilities are based on a parallel architecture. There are different kind of electronic implementation of ANN, digital, analog and hybrid (M.A. Banuelos, j. Castillo Hernandez, S. Quintana Thierry, R. Damian Zamacoma, J. Valeriano Assem, R.E. Cervantes, R. Fuentes Conzalez, G. Calva Olmos, J.L. Perez Silva, 2003) and each one has specific advantage and disadvantages depending on the type and configuration of the network, training method and application.

For a digital implementation of ANN there are different alternatives, custom design, digital signal processors, programmable logic, etc. Programmable logic offers low cost, powerful software tools and true parallel implementations. Field Programmable Gate Arrays $\left(\right.$ FPGA $_{\mathrm{s}}$ ) consist in a family of a programmable logic devices based in an array of configurable logic blocks, which gives a great flexibility in the development of digital systems. The amount of their available resources for digital system design gives a great flexibility for implementing comp lex systems, such as a specific purpose microprocessor, network controllers, digital video systems, and for implementing digital ANNs. (Bogdan M. Wilamon wski, 2009).

* Corresponding author 
In this paper the authors present the design of an artificial neuron on the ALTERA $^{\circledR}$ Cyclone III EP3C25F324C8 FPGA evaluation board. In general a neuron comprises an input aggregation stage and an output activation function one.

The input stage of implemented neuron is based on a sfloat 24 adder. Fundamentally three types of activation functions have been used: step, ramp-saturation, and sigmoid. For implementing the sigmoid activation function the well known CORDIC (Ray Andraka, 1998) theory is used. The obtained results will be used, as a starting point, for the generation of complex ANN for application requiring parallel computing.

The authors was developed a floating point math library for FPGAs, called sfloat24 (M.C. Miglionico, F. Parillo, 2010), which is used in this paper for the Artificial Neuron implementation. Respect to the classical IEEE 754 (IEEE Standard for Binary Floating-Point Arithmetic, 1985) (W. Kahan, 1996) floating point number format, the numbers are represented by 24 bit word length. This reduced representation is useful when a small FPGA is used.

The implementation of the Artificial Neuron has been based on the definition of the basic arithmetic operations and of the comparison operator (C. Attaianese, F. Parillo, G. Tomasso, 2010), successively applying the CORDIC theory in order to define the exponential function.

\section{Modelling of an Artificial Neuron}

A generic neuron, as well known, consist of several weighted inputs, an adder function and an activation function as shown in Figure 1.

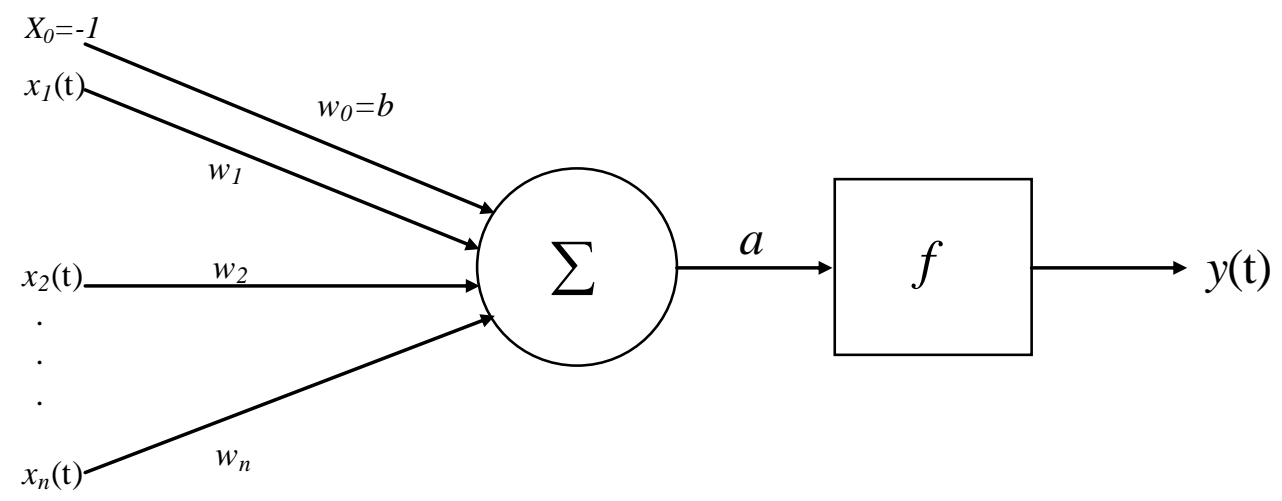

Figure 1. Block diagram of an artificial neuron.

where $y(t)$ is the output of the neuron. $\mathrm{W}$ is the input vector, $x_{i}(t), i \in[0 ; n]$ are the inputs and $f$ is a non linear activation function. This neuron model correspond to the following generalization:

$$
y=f(a)=f\left(\sum_{i=0}^{n} w_{i} \cdot x_{i}\right)=f(W \otimes X)
$$

with $X=\left[\begin{array}{c}x_{0} \\ x_{1} \\ x_{2} \\ \cdot \\ \cdot \\ x_{n}\end{array}\right]$ and $W=\left[\begin{array}{c}w_{0} \\ w_{1} \\ w_{2} \\ \cdot \\ \cdot \\ w_{n}\end{array}\right]$

where: $x_{0}$ is equal to -1 and $w_{0}=b$, where $b$ is a bias and/or a threshold. 
The simplest activation function is the step activation function, defined as follow:

$$
y(a)= \begin{cases}0 & \text { if } a<\theta \\ 1 & \text { if } a>=\theta\end{cases}
$$

where $a$, see Figure 1, is the sum of all input signal and $\theta$ is a threshold level.

Another used activation function is the ramp saturation activation function that produces an output gradually growing from zero to a fixed maximum level. This function can be expressed as:

$$
y(a)= \begin{cases}a & \text { if } a<\theta \\ \vartheta & \text { if } a>=\theta\end{cases}
$$

where $\theta$ is the saturation level.

The back-propagation (BP) learning process is widely adopted as a successful learning rule to find the appropriate values of the weights for ANNs. For example the Multi-Layer networks consists of various layers: an input and output ones between which lie one or several hidden ones whose outputs are not observable. These layers are based upon some processing unit (neurons) interconnected by means of feedforward pondered links as depicted in Figure 2. (Mellit, S. Shaari, H. Mekki, N. Khorissi, 2008). The BP learning process requires that the activation function is derivable. An example of a derivable function is the sigmoid.

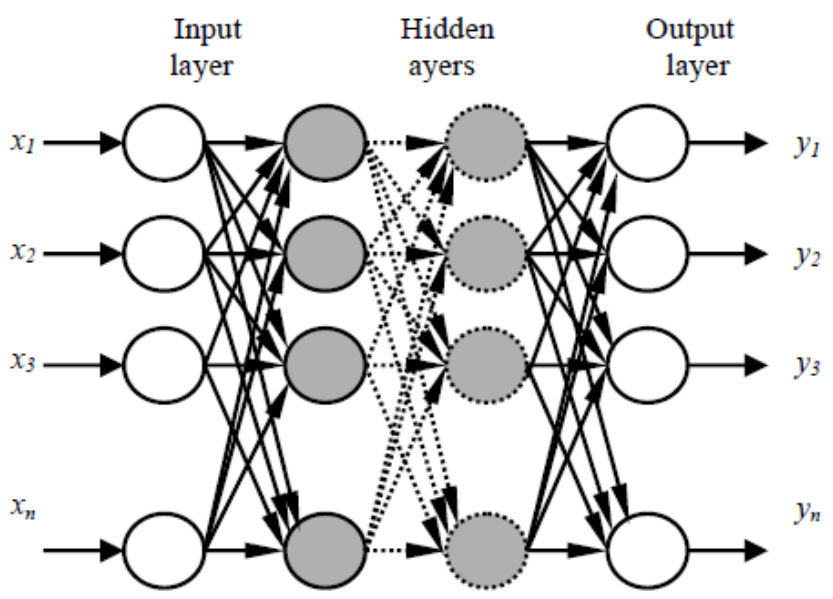

Figure 2. Schematic diagram of a multi-layer feed-forward neural network.

A sigmoid function has the following expression:

$$
f(x)=\frac{1}{\left(1+e^{-x}\right)}
$$

This function has been implemented by means the developed custom sfloat 24 math library and using the CORDIC theory for the implementation of the exponential function.

Various methods exist to implement a trigonometric/hyperbolic math function. These include Taylor series; various curve fitting algorithms and the CORDIC (Oordinate Rotation DIgital Computer) algorithm. The CORDIC algorithm often offers the most elegant solution to the problem, and it is astounding in its simplic ity of implementation, efficiency and elegance (Samuel Ginsberg, 2002).

The CORDIC algorithm provides an iterative method of performing vector rotations by arbitrary angles using only shift and adds. Usually the CORDIC theory is used to implement trigonometr ic functions. The implementation of the Sine and Cosine functions is quite immediate. 
Applying this theory and assuming 12 step system, this yields 12 bits of accuracy in the final answer. Note that the Konst $=\operatorname{Cos} \vartheta$, constant for a 12 step algorithm, is given by:

$$
K=\prod_{i=0}^{11} \cos \theta_{i}=0.6075
$$

where:

$$
\theta_{i}=\tan ^{-1}\left(2^{-i}\right)
$$

At begin, off line stage, has been built up two small look up tables, in this case with a length of 12 elements, in the one is stored the angles in power of 2 , starting from $\vartheta[0]=\frac{\pi}{4}$, and in the other the reciprocal of the power of 2 . The second look up table is useful to avoid the divide by 2 operation during the execution of the algorithm (real time stage).

The first look up table is denoted fract_teta[12], where the stored angles are in radiants, and the second reciprocal_pow2[12]. In the following is depicted a fragment of $\mathrm{C} / \mathrm{C}++$ code to implement the sine and cosine functions.

Initialisation:

Set $\vartheta$, 'teta_rad' is the input angle, expressed in radians;

set $y=0$;

set $x=$ Konst $=0.60725$;

Computation:

$y=0.0$

$d x=0.0 ; d y=0.0$;

for $(i=0 ; i<12 ; i++)$

\{

$d x=x *$ reciprocal_pow $2[i]$;

$d y=y^{*}$ reciprocal_pow2 [i];

dteta_rad=fract_teta[i];

if $($ teta_rad $>=0) d=-1.0$; else $d=1.0$;

$x=x+d^{*} d y$;

$y=y-d^{*} d x$;

teta_rad=teta_rad $+d^{*} d t e t a \_r a d$;

\}

The trigonometric functions of the desired angle are present in the $\mathrm{x}$ and $\mathrm{y}$ variable. In this implementation is assumed $-\frac{\pi}{2}<\vartheta<\frac{\pi}{2}$ in order to extend the computation for any value of $\vartheta$ it is sufficient to add few instructions, as well known in the literature.

In the case of the implementation of the hyperbolic function the expressions, us ing always the CORDIC theory, can be written as follow:

$$
\begin{aligned}
& \vartheta_{i+1}=\vartheta_{i}-d_{i} \tanh ^{-1}\left(2^{-i}\right) \\
& x_{i+1}=x_{i}+y_{i} d_{i} 2^{-i} \\
& y_{i+1}=y_{i}+x_{i} d_{i} 2^{-i} \\
& \vartheta_{i+1}=\vartheta_{i}-d_{i} \tanh ^{-1}\left(2^{-i}\right)
\end{aligned}
$$

where $d_{i}=-1$ if $\vartheta_{i}<0,+1$ otherwise, the integer $i$ varies as described below. 
The hyperbolic functions, considering the expressions (5) and (6), can be implemented in similar manner as the sine and cosine functions. In fact, the follow ing code is quite similar:

Initialisation:

Set $\vartheta$, 'teta_rad' is an input variable;

set $y=0$;

set $x=$ Konst $=7.5495 e+011$;

Computation:

$y=0.0$;

$d x=0.0 ; d y=0.0$;

for $(i=0 ; i<24 ; i++)$

\{

$d x=x *$ reciprocal_pow2 $[i]$;

$d y=y^{*}$ reciprocal_pow $2[i]$;

dteta_rad=fract_teta[i];

if $($ teta_rad $>=0) d=-1.0$; else $d=1.0$;

$x=x+d^{*} d y$

$y=y+d^{*} d x$

teta_rad=teta_rad-d*dteta_rad;

\}

reciprocal_pow2[i] is given by:

$$
\begin{aligned}
& / *\left[1-2^{-13}, 1-2^{-12}, 1-2^{-11}, 1-2^{-10}, 1-2^{-9}, 1-2^{-8}, 1-2^{-7}, 1-2^{-6}, 1-2^{-5}, 1-2^{-4}, 1-2^{-3}, 1-2^{-2},\right. \\
& \left.2^{-1}, 2^{-2}, 2^{-3}, 2^{-4}, 2^{-5}, 2^{-6}, 2^{-7}, 2^{-8}, 2^{-9}, 2^{-10}, 2^{-11}, 2^{-12}\right]^{*} /
\end{aligned}
$$

and fract_teta $[i]$ is given by the inverse hyperbolic tangent of the above commented values.

The hyperbolic functions are present in the $\mathrm{x}$ and $\mathrm{y}$ variables. In this case the steps are 24 because the input variable can assume any value, the hyperbolic are not circular math functions.

At last the exponential functions can be easily derived from the hyperbolic functions as following:

$$
e^{x}=\sinh (\vartheta)+\cosh (\vartheta) \quad e^{-x}=-(\sinh (\vartheta)+\cosh (\vartheta)
$$

\section{FPGA Implementation - Simulation resul ts -}

It is possible, on the basis of expressions (5),(6) and (7), to build the sigmoid function. The code of the hyperbolic functions has been written in VHDL code, similar at above depicted C code.

The code has been implemented using the ALTERA ${ }^{\circledR}$ Quartus II 9.1 software. The sfloat 24 sigmoid activation function occupies only $44 \%$ of total logic elements of the used Cyclone ${ }^{\circledR}$ III EP3C25F324C8 device and occupying under $1 \%$ of the dedicated logic registers.

In the following is shown the obtained sigmoid function compared to the same function built with Simulink ${ }^{\circledR}$ blocks. It is important to underline that the Matlab ${ }^{\circledR}$ operates with double precis ion floating (64 bit) point numbers. The simulations have been performed using the ALTERA ${ }^{\circledR}$ DSP Builder tool, in particular using the HDL import block, as depicted in Figure 3.

Figure 4 shows the simulation results when the sampling time $T_{s}$ has been fixed to a $2.56 \mu \mathrm{S}$, in this case the output error varies in the range \pm 0.006 about. Other simulations have been performed with different sampling times. In all the tests results that the entire system has a latency time at maximum of 6 clock cycles. Improvement of the overall performances could be reached by using a sample time less than 2.56 $\mu \mathrm{S}$. For a common industrial application the used sample time produces good results. 


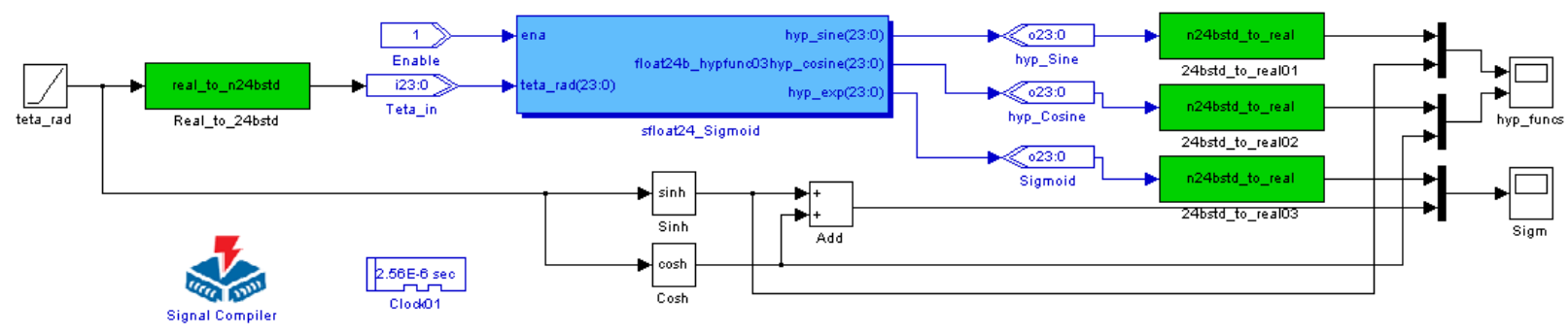

Figure 3. Hyperbolic and Sig moid functions Simu lin $\mathrm{k}^{(} \operatorname{simulation~layout.~}$
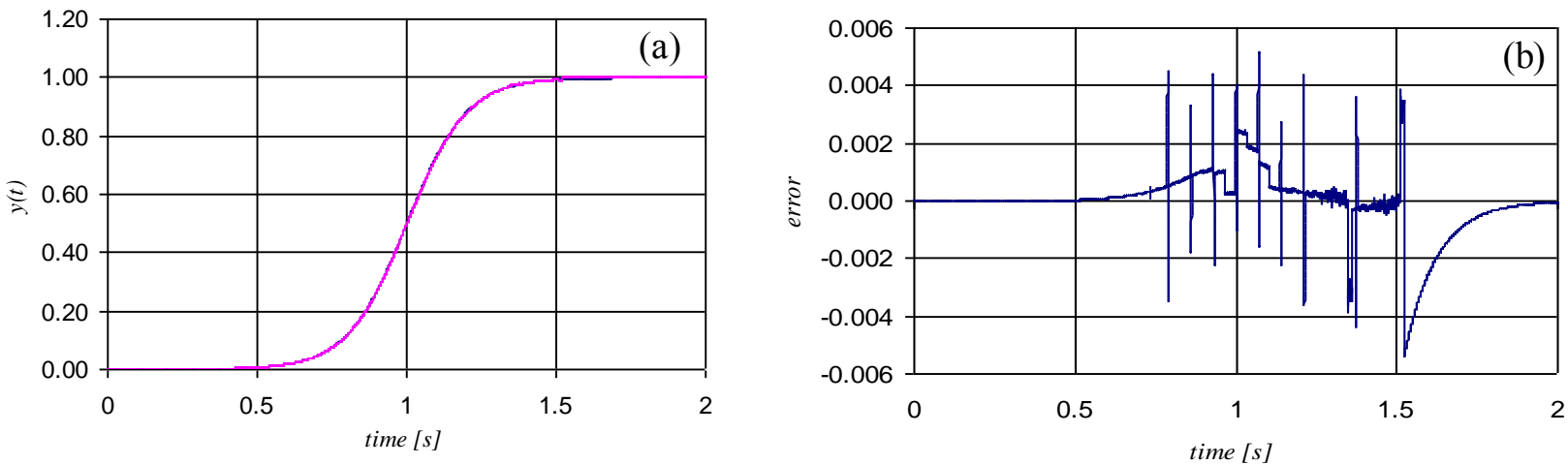

Figure 4. Simulation results - (a) Sig mo id activation function and (b) the absolute error between Sig moid generated by the sfloat 24 math library and one generated by Simulink ${ }^{\circledR}$ math function block.

The system presents a stable performance comparing to the any external disturbance. In fact, FPGA offer the stability of a typical digital implementation on a standard microprocessor and performances comparable to an analog implementation. Two additional routines allow to test the implemented ANN performances. The first routine converts a given floating point number in to sfloat 24 number layout format. The second one converts a sfloat 24 number to a double precision floating number.

These routine, due to the ir complexity, have been written in $\mathrm{C} / \mathrm{C}++$ language and implemented as $\mathrm{S}$ Function as depicted in (M.C. Miglionico, F. Parillo, 2010).

\section{Conclusions}

The obtained results show the validity and the feasibility of implementing a Sigmoid activation function using either the custom sfloat 24 math library and the CORDIC theory. On the used FPGA device is possible only to implement a single artificial neuron. These results show that it is possible implement a full Artificial Neural Network similar the one shown in Figure 2. In this case a ALTERA ${ }^{\circledR}$ Stratix FPGA is required. The authors are working on an optimized version of the proposed algorithm, in order to reduce the area device occupation, in terms of logic elements, on any used FPGA. Furthermore, the speed or execution or latency of the ANN can be precisely controlled with the amount of reuse of sfloat 24 arithmetic elements.

Since on the more advanced families of FPGAs it is possible to implement a network with an interesting number of neurons working in parallel using only a single chip.

The implemented sigmoid module is highly accurate and can easily be reconfigured for any sigmoid slope and any desired error tolerance. 


\section{REFERENCES}

M.A. Banuelos, j. Castillo Hernandez, S. Quintana Thierry, R. Damian Zamacoma, J. Valeriano Assem, R.E. Cervantes, R. Fuentes Conzalez, G. Calva Olmos, J.L. Perez Silva. (2003). Implementation of a Neuron Using FPGAS. Journal of Applied Research and Technology, Vol. I numero 003, 3 October 2003, Universitad Nacional Autonoma de Mexico Distrito Federal, Mexico, $248-255$.

Bogdan M. Wilamonwski. (2009). Neural Network Architectures and Learning Algorithms. IEEE Industrial Electronics Magazine, December 2009.

Ray Andraka. (1998). A survey of CORDIC algorithms for FPGA based computers. In: International Symposium on Field Programmable Gate Arrays, Monterey, California, United States 1998 , 191 - 200.

M.C. Miglionico, F. Parillo. (2010). FPGA implementation of sfloat24 digital PI. IEEE Conference PEDES 2010 Power India, New Delhi, 20-23 December 2010.

IEEE Standard for Binary Floating-Point Arithmetic. (1985). ANSI/IEEE 7541985.

W. Kahan. (1996). Lecture notes on the Status of IEEE Standard 754 for Binary Floating Point Arithmetic. Electrical Engineering and Computer Science - University of California Berkeley CA 94720-1776, 31 May 1996.

C. Attaianese, F. Parillo, G. Tomasso. (2010). Dual Boost High Performances control strategy on a Power Factor Correction (PFC) implementation by us ing a 24 bit custom floating point library. Journal of Electrical Engineering [http://www.jee.ro], Vol. 10 edition 4, 23 Dec ember 2010.

Mellit, S. Shaari, H. Mekki, N. Khorissi. (2008). FPGA-based Artificial Neural Network for Prediction of Solar Radiation Data from Sunshine Duration and Air Temperature. Proceedings 2008 IEEE Region 8 International Conference on Computational technologies in Electrical and Electronic engineering. “SIBIRCON 2008", 118-123.

Samuel Ginsberg. (2002). Compact and Efficient Generation of Trigonometric Functions using a CORDIC algorithm. Cape Town, South Africa, January 2002. 